\title{
Nowcast and forecast of galactic cosmic ray (GCR) and solar energetic particle (SEP) fluxes in magnetosphere and ionosphere - Extension of WASAVIES to Earth orbit
}

\author{
Tatsuhiko Sato ${ }^{1, *}$, Ryuho Kataoka ${ }^{2,3}$, Daikou Shiota ${ }^{4,5}$, Yûki Kubo ${ }^{4}$, Mamoru Ishii ${ }^{4}$, Hiroshi Yasuda ${ }^{6}$, \\ Shoko Miyake ${ }^{7}$, Yoshizumi Miyoshi ${ }^{5}$, Haruka Ueno $^{8}$, and Aiko Nagamatsu ${ }^{8}$ \\ ${ }^{1}$ Japan Atomic Energy Agency (JAEA), Shirakata 2-4, Tokai, Ibaraki 319-1195, Japan \\ 2 National Institute of Polar Research (NIPR), Midori-cho, Tachikawa, Tokyo 190-8518, Japan \\ ${ }^{3}$ SOKENDAI, Midori-cho, Tachikawa, Tokyo 190-8518, Japan \\ ${ }^{4}$ National Institute of Information and Communications Technology (NICT), Nukui-Kitamachi 4-2-1, Koganei, Tokyo 184-0015, Japan \\ ${ }^{5}$ Nagoya University, Furo-cho, Chikusa-ku, Nagoya 464-8601, Japan \\ ${ }^{6}$ Hiroshima University, Kasumi 1-2-3, Minami-ku, Hiroshima 734-8553, Japan \\ ${ }^{7}$ National Institute of Technology, Ibaraki College, Nakane 866, Hitachinaka, Ibaraki 312-8508, Japan \\ ${ }^{8}$ Japan Aerospace Exploration Agency (JAXA), Sengen 2-1-1, Tsukuba, Ibaraki 305-8505, Japan
}

Received 7 September 2018 / Accepted 15 February 2019

\begin{abstract}
Real-time estimation of cosmic-ray fluxes on satellite orbits is one of the greatest challenges in space weather research. Therefore, we develop a system for nowcasting and forecasting the galactic cosmic ray $(\mathrm{GCR})$ and solar energetic particle (SEP) fluxes at any location in the magnetosphere and ionosphere during ground-level enhancement (GLE) events. It is an extended version of the WArning System for AVIation Exposure to SEP (WASAVIES), which can determine event profiles by using real-time data of the count rates of several neutron monitors (NMs) at the ground level and high-energy proton fluxes observed by Geostationary Operational Environmental Satellites (GOES) satellites. The extended version, called WASAVIES-EO, can calculate the GCR and SEP fluxes outside a satellite based on its two-line element (TLE) data. Moreover, organ absorbed-dose and dose-equivalent rates of astronauts in the International Space Station (ISS) can be estimated using the system, considering its shielding effect. The accuracy of WASAVIES-EO was validated based on the dose rates measured in ISS, as well as based on high-energy proton fluxes observed by POES satellites during large GLEs that have occurred in the $21 \mathrm{st}$ century. Agreement between the nowcast and forecast dose rates in ISS, especially in terms of their temporal structures, indicates the usefulness of the developed system for future mission operations.
\end{abstract}

Keywords: SEP / dosimetry / ground-level enhancement (GLE) / radiation protection / space weather

\section{Introduction}

Estimation of the fluxes of high-energy particles in the Earth's magnetosphere is very important for designing space missions because they can adversely affect the health of astronauts and cause single-event upsets of semi-conductor devices used in satellites. Three sources must be considered in the estimation, namely, galactic cosmic rays (GCRs), trapped particles (TPs), and solar energetic particles (SEPs). The fluxes of GCR and TP are relatively stable and predictable compared to that of SEP, and the procedures for calculating GCR (Nymmik et al., 1996; Matthiä et al., 2013; O'Neill et al.,

*Corresponding author: sato.tatsuhiko@jaea.go.jp
2014; Slaba \& Blattnig, 2014) and TP (Ginet et al., 2013) fluxes are rather well established. In addition, software for calculating their mean fluxes in certain Earth orbits, such as CREME96 (Tylka et al., 1997) and SPENVIS (Heynderickx et al., 2004), were developed and released to public.

By contrast, SEP fluxes during a space mission are unpredictable because they increase suddenly when a large solar particle event (SPE) occurs. Thus, the worst-case scenarios are generally considered in mission design (Xapsos et al., 1999; Aghara et al., 2015; Jiggens et al., 2018). On the other hand, estimation of real-time SEP fluxes during SPE is beneficial for mission operation to take adequate actions, such as sheltering astronauts in well-shielded locations in their spacecraft (Townsend et al., 2018). A few studies (Hu et al., 2016; 


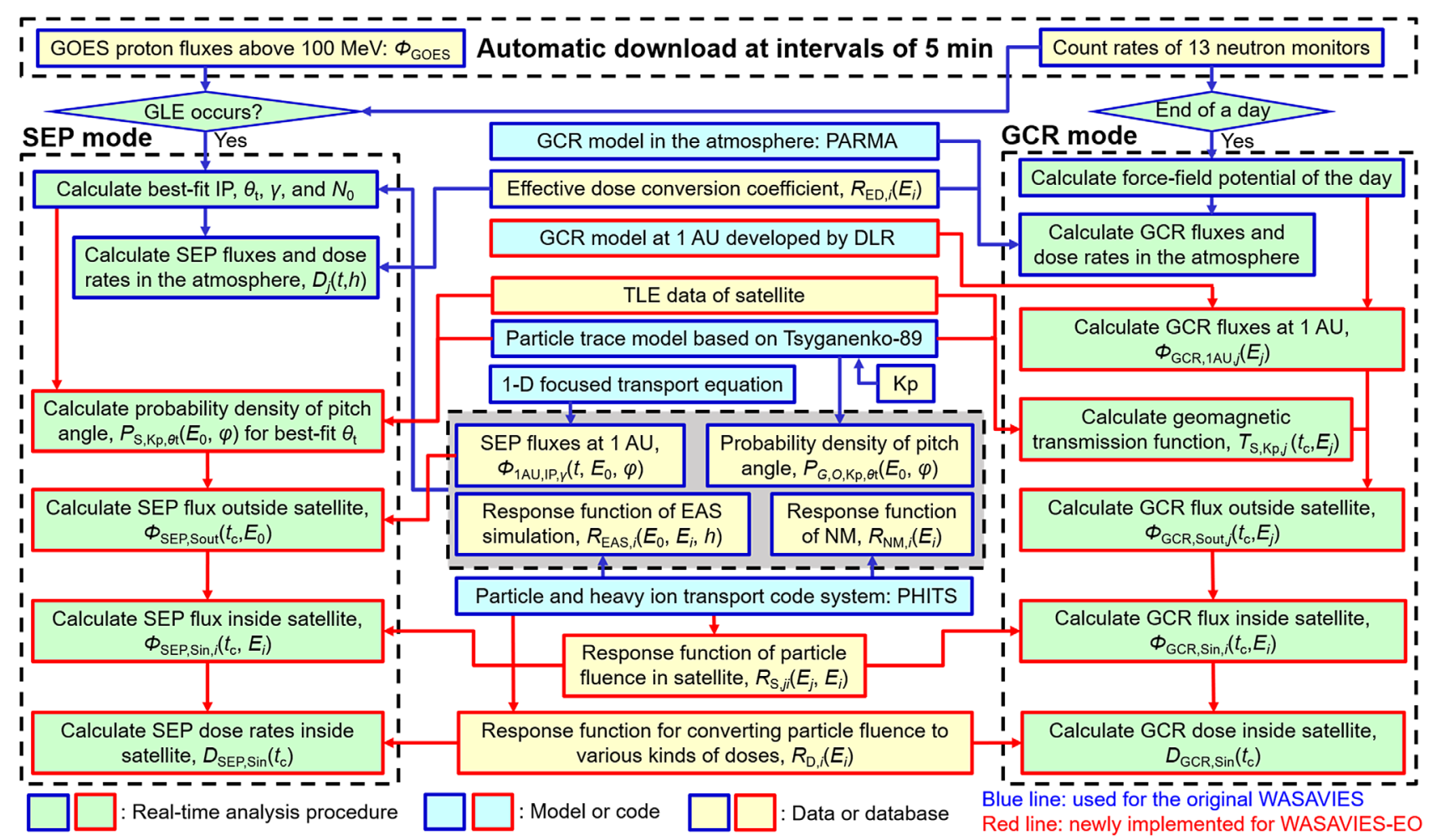

Fig. 1. Flowchart of calculation procedures of WASAVIES-EO. The pastel green, cyan, and yellow boxes indicate the real-time analysis procedure, model or code, and data or database, respectively. The boxes in the blue frame are used in the original WASAVIES, while those with red frame are newly implemented in WASAVIES-EO. All databases included in the gray box are used for calculating the best-fit IP, $\theta_{t}, \gamma$, and $N_{0}$, while only the database of SEP fluxes at $1 \mathrm{AU}$ is used for calculating SEP flux outside satellite. The Kp index is automatically updated at intervals of $3 \mathrm{~h}$.

Matthiä et al., 2018) have been conducted to reproduce the SEP fluxes in spacecraft during certain SPEs, but these studies were mostly dedicated to post-exposure evaluation. Therefore, it is desirable to develop a system that can nowcast or forecast the SEP fluxes during a SPE. An active dosimeter-based method for estimating astronaut acute radiation risk during SPE in real time was recently proposed (Mertens et al., 2018).

With these situations in mind, we set out to develop a new computational method that can nowcast and forecast SEP fluxes at any location in the magnetosphere and ionosphere during a large SPE associated with a ground-level enhancement (GLE). It is an extended version of the WArning System for AVIation Exposure to SEP (WASAVIES) (Kataoka et al., 2014; Kataoka et al., 2018; Sato et al., 2018b), which can nowcast and forecast radiation doses in the atmosphere by using real-time data of the count rates of several neutron monitors (NMs) at the ground level and high-energy proton fluxes observed by Geostationary Operational Environmental Satellites (GOES). The contribution of GCR can be also calculated by the system by using the PARMA model (Sato, 2015, 2016). The most important feature of WASAVIES is that it is fully based on physics models of SEP transport from the Sun to the ground level of the Earth, and this feature enables us to smoothly extend the system applicable to the magnetosphere and ionosphere.

The extended system is called WASAVIES-EO, where EO represents Earth Orbit. The two-line element (TLE) data of a satellite must be supplied to the system for calculating the
GCR and SEP fluxes on its orbit. The organ doses and dose equivalents of astronauts in International Space Station (ISS) can be also estimated by the system considering its shielding effect, which has been evaluated using the Particle and Heavy Ion Transport code System (PHITS, Sato et al., 2018a) coupled with a virtual ISS model. Detailed calculation procedures of the original WASAVIES can be found in our previous paper (Sato et al., 2018b). Thus, the present study focuses on describing the extended part of the calculation procedures, together with its validation results, based on the experimental data measured by ISS and Polar Orbiting Environmental Satellites (POES) during large GLEs that have occurred in the 21 st century.

\section{Calculation procedures}

Figure 1 shows a flowchart of the calculation procedures of the system developed in this study. The algorithm employed in the original WASAVIES is summarized briefly in Section 2.1, while that of the extended part is described in detail in Sections 2.2 and 2.3 .

\subsection{Basic algorithm of WASAVIES}

In WASAVIES, the profiles of each SPE are characterized by four parameters, namely, injection profile (IP), power index $\gamma$ of the primary SEP around the Sun, north-south tilt angle of 
ambient magnetic field incident to the magnetosphere $\theta_{t}$, and normalization coefficient of the SEP fluence $N_{0}$. These parameters are determined automatically from the count rates of several NMs at the ground level and the proton fluxes measured by GOES, which are downloaded continuously at intervals of $5 \mathrm{~min}$. Note that we adopted the power law spectrum in rigidity of the primary SEP around the Sun.

Four databases must be prepared before executing the realtime and automatic analysis program of WASAVIES, and they are as follows: 1 . Time profile of primary SEP fluxes at 1 astronomical unit (AU) for a certain IP and $\gamma, \Phi_{1 \mathrm{AU}, \mathrm{IP}, \gamma}\left(t, E_{0}, \varphi\right)$, where $t$ is the time after flare onset, $E_{0}$ is energy of the primary $\mathrm{SEP}$, and $\varphi$ is pitch angle incident to the magnetosphere from the ambient magnetic field, the so-called Parker spiral's interplanetary magnetic field defined here as $45^{\circ}$ declined from the Sun-Earth line. 2. The probability densities of the pitch angle $\varphi$ of protons with energy $E_{0}$ that can penetrate through the magnetosphere, $P_{G, O, \mathrm{Kp}, \theta_{t}}\left(E_{0}, \varphi\right)$, where $G$ indicates the geographic coordinate of the arrival location at the top of the atmosphere, $O$ denotes Earth's orbital condition characterized by month and hour, $\mathrm{Kp}$ is $\mathrm{Kp}$ index. 3. The response function of the fluences of particle $i$ with energy $E_{i}$ in the atmosphere generated through extensive airshowers (EAS) induced by mono-energetic protons with energy $E_{0}, R_{\mathrm{EAS}, i}\left(E_{0}, E_{i}, h\right)$, where $h$ denotes altitude. 4. The response functions of the standard NM64 NM for the incidence of particle type $i$ with energy $E_{i}, R_{\mathrm{NM}, i}\left(E_{i}\right)$.

The $\Phi_{1 \mathrm{AU}, \mathrm{IP}, \gamma}\left(t, E_{0}, \varphi\right)$ database was developed by solving one-dimensional focused transport equations by using the method developed by Kubo et al. (2015), while $P_{G, O, \mathrm{Kp}, \theta_{t}}$ $\left(E_{0}, \varphi\right)$ was prepared by tracing the trajectories of anti-protons emitted from the top of the atmosphere by using the proton trace model (Miyake et al., 2017) based on the empirical geomagnetic field model T89 (Tsyganenko, 1989). The other databases were constructed by performing Monte Carlo simulations by using the PHITS version 2.88 (Sato et al., 2018a). Details of the PHITS simulation procedures can be found in Sato et al. (2014).

In the real-time analysis program, GOES proton fluxes above $100 \mathrm{MeV}$ and the count rates of 13 selected NMs are downloaded automatically from the Space Weather Prediction Center of NOAA (ftp://ftp.swpc.noaa.gov/pub/lists/particle/) and the Neutron Monitor Database NMDB (http://www.nmdb. $\mathrm{eu} /$ ), respectively, at 5-min intervals. Information about the selected NM stations can be found in our previous paper (Sato et al., 2018b). At the end of each day, the daily count rates of each NM are calculated and used for determining the force field potential of the day. The GCR fluxes and the corresponding dose rates in the atmosphere are then calculated using the PARMA model (Sato, 2015, 2016) coupled with the evaluated force field potential.

The occurrence of GLE is checked by comparing the background and the current data of the NM count rates, as well as the GOES proton fluxes (Sato et al., 2018b). When the program detects GLE, it activates the SEP mode and automatically determines the best-fit values of the four parameters, namely, IP, $\gamma, \theta_{t}$, and $N_{0}$, to reproduce the NM count rates and GOES proton fluxes. The SEP fluxes and the corresponding dose rates in the atmosphere are then calculated in real time by using the best-fit parameters. In parameter determination and dose evaluation, the aforementioned four databases, as well as the database of the effective dose conversion coefficients for isotropic irradiation
(ICRP, 2010), are used. In addition, WASAVIES can forecast the SEP dose rates up to $24 \mathrm{~h}$ after flare onset, assuming that the evaluated parameters are time-independent, i.e., the time variation of the future SEP fluxes at $1 \mathrm{AU}$ exactly follows their pre-calculated data contained in the database of $\Phi_{1 \mathrm{AU}, \mathrm{IP}, \gamma}\left(t, E_{0}, \varphi\right)$. However, this assumption is occasionally not adequate because the power index of the primary SEP, $\gamma$, varies with time for some events, as discussed in our previous paper (Sato et al., 2018b).

\subsection{Preparation of databases used in WASAVIES-EO}

Two databases were additionally prepared for extending WASAVIES for application to Earth orbits, and they are as follows: 1. Response function of the fluences of particle $i$ with energy $E_{i}$ at certain locations in a satellite for isotropic irradiation of mono-energetic particles $j$ with energy $E_{j}, R_{S, j i}\left(E_{j}, E_{i}\right)$. 2 . Response functions for converting the fluence of particle $i$ with energy $E_{i}$ to various types of doses, $R_{D, i}\left(E_{i}\right)$.

For preparing $R_{S, j i}\left(E_{j}, E_{i}\right)$, we performed a cosmic-ray transport simulation by using PHITS coupled with a virtual ISS model developed by Japan Aerospace Exploration Agency (JAXA) (Sato et al. 2018c). Figure 2 shows a three-dimensional (3D) view of the virtual ISS model drawn using PHITS. Only the Kibo, Columbus, Harmony, and Destiny modules have been modeled thus far. The models are composed mainly of the walls and the payload racks of these modules, which are made of $\mathrm{Al}$ alloys and $\mathrm{Al}$, respectively, and their masses are approximated to those of the real ones. Protons and ions with energies up to $1 \mathrm{TeV} / \mathrm{u}$ and charges up to $28(\mathrm{Ni})$ were considered as source particles $j$, and the fluences of those particles, as well as neutrons, pions, electrons, positrons, and photons, at several locations in the Kibo module were scored in the simulation. In this study, $R_{S, j i}\left(E_{j}, E_{i}\right)$ inside the pressurized module of Kibo was used to validate the accuracy of WASAVIES-EO. The mean shielding thickness of the module was estimated to be $35.6 \mathrm{~g} \mathrm{~cm}^{-2}$.

For $R_{D, i}\left(E_{i}\right)$, we prepared the response functions of two silicon-based active detectors-Liulin-5 in the MATROSHKA$\mathrm{R}$ phantom and DOSTEL (DOSimetry TELescope)- to reproduce the experimental data presented by Reitz et al. (2005), Semkova et al. (2014), and Berger et al. (2018). The response function of Liulin-5 was evaluated by performing the PHITS simulation for mono-energetic particle irradiation incident on the MATROSHKA-R phantom-a spherical tissue-equivalent material with a radius of $17.5 \mathrm{~cm}$ - and scoring the absorbed dose around the location of Liulin-5 used for our validation, that is, at a depth of $4 \mathrm{~cm}$ depth from the surface. By contrast, the response function of DOSTEL was assumed to be the same as the stopping power of silicon because of the thin thickness of the detector-315 $\mu \mathrm{m}$. In addition, fluence to dose and $Q(L)$-based dose-equivalent conversion coefficients for red bone marrow (RBM) of the reference adult male phantom were included in the database, where their numerical values were taken from the International Committee on Radiological Protection (ICRP) Publications 116 and 123 (ICRP, 2010, 2013).

\subsection{Extension to real-time analysis program}

The extended part of the real-time analysis program in WASAVIES-EO is executed after all analyses processes in 


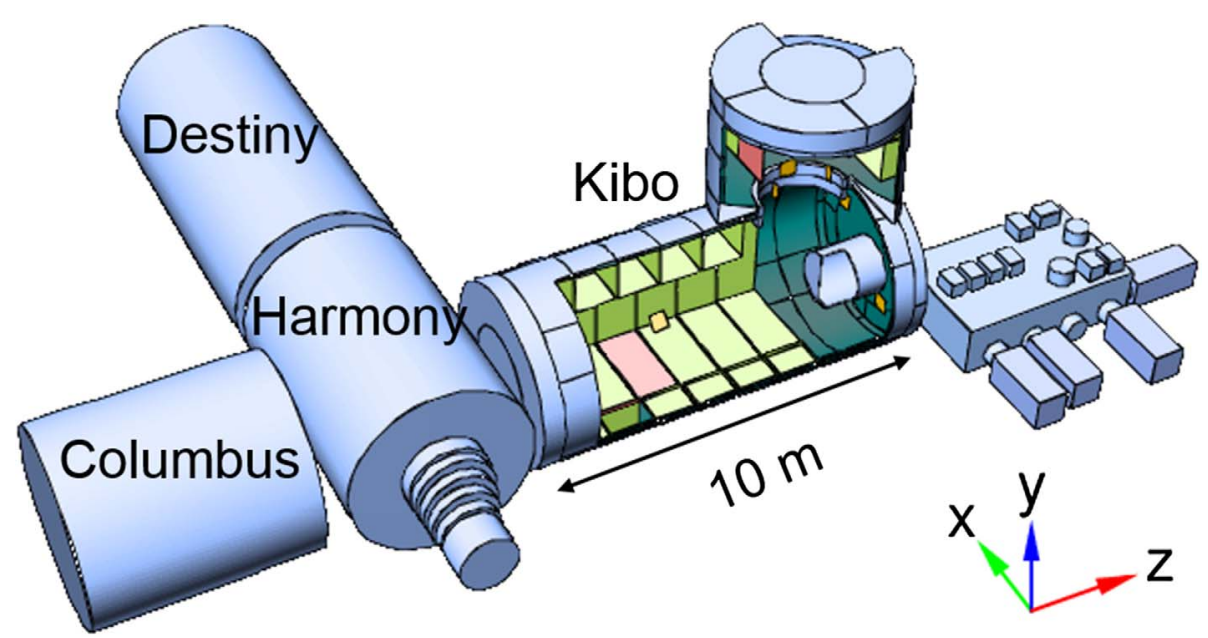

Fig. 2. 3D view of virtual ISS model drawn using PHITS. A cut-away section of Kibo module is presented to show its interior structure.

the original WASAVIES are completed. During the solar quiet period, at first, the GCR fluxes of particle $j$ with energy $E_{j}$ outside the magnetosphere, $\Phi_{\mathrm{GCR}, 1 \mathrm{AU}, j}\left(E_{j}\right)$, are calculated using the GCR model developed by Matthiä et al. (2013) coupled with the daily force field potential evaluated from the count rates of the selected $\mathrm{NM}$ monitors. Note that $\Phi_{\mathrm{GCR}, 1 \mathrm{AU}, j}\left(E_{j}\right)$ is assumed to be constant for a day, even during GLE, although the actual GCR fluxes fluctuate slightly if Forbush decrease occurs. This assumption indicates that the SEP contributions to the NM count rates and the GOES proton fluxes during GLE can be simply determined from the difference between their real-time and background data, where the background data were evaluated before the occurrence of GLE. Then, the geomagnetic transmission function of particle $j$ with energy $E_{j}$ at the satellite location at the current time $t_{c}, T_{S, \mathrm{Kp} . j}\left(t_{c}, E_{j}\right)$, is calculated using the particle trace model (Miyake et al., 2017) based on the T89 model (Tsyganenko, 1989). The TLE data of a satellite should be supplied by a user for this calculation, where the data for most satellites can be downloaded from the website of Space Track (https://www.space-track.org). Note that TLE is a de facto standard data format encoding a list of orbital elements. The GCR fluxes of particle $j$ outside the satellite at $t_{c}$,

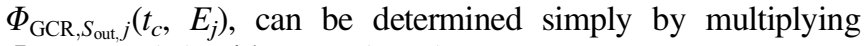
$\Phi_{\mathrm{GCR}, 1 \mathrm{AU}, j}\left(E_{j}\right)$ with $T_{S, \mathrm{Kp}, j}\left(t_{c}, E_{j}\right)$.

During GLE, the probability densities of the pitch angle $\varphi$ of protons with energy $E_{0}$ that can penetrate through the magnetosphere to the satellite location at $t_{c}$ for the best-fit $\theta_{t}$, $P_{S, \text { Kp }, \theta_{t}}\left(t_{c}, E_{0}, \varphi\right)$, are calculated using the same particle trace model and TLE data. In this calculation, the program traces the trajectory of anti-protons with energy $E_{0}$ emitted from the satellite location in 288 directions, covering a solid angle of $4 \pi$, and estimates their pitch angle $\varphi$ escaping from the magnetosphere, where $\varphi$ is determined from the direction of the ambient magnetic field and the asymptotic direction. The current SEP fluxes outside the satellite, $\Phi_{\mathrm{SEP}, S_{\text {out }}}\left(t_{c}, E_{0}\right)$, are calculated using the following equation:

$$
\begin{aligned}
\Phi_{\mathrm{SEP}, S_{\text {out }}}\left(t_{c}, E_{0}\right)= & \int N_{0}\left(t_{c}\right) \Phi_{1 \mathrm{AU}, \mathrm{IP}, \gamma}\left(t_{c}-t_{0}, E_{0}, \varphi\right) \\
& \times P_{S, \mathrm{Kp}, \theta_{\mathrm{t}}}\left(t_{\mathrm{c}}, E_{0}, \varphi\right) \mathrm{d} \varphi
\end{aligned}
$$

where $N_{0}\left(t_{c}\right)$ is the normalization coefficient of the SEP fluxes calculated using WASAVIES, $\Phi_{1 \mathrm{AU}, \mathrm{IP}, \gamma}\left(t, E_{0}, \varphi\right)$ is the time profile of the primary SEP fluxes at $1 \mathrm{AU}$ for the best-fit IP and $\gamma$, and $t_{c}-t_{0}$ is the time since flare onset. Note that the lowest value of energy $E_{0}$ included in the $\Phi_{1 \mathrm{AU}, \mathrm{IP}, \gamma}\left(t, E_{0}, \varphi\right)$ database is $80 \mathrm{MeV}$, which is sufficiently low to estimate aircrew doses but excessive for estimating astronaut doses. Thus, the program extrapolates the SEP fluxes down to $10 \mathrm{MeV}$ by using the power index of $\Phi_{1 \mathrm{AU}, \mathrm{IP}, \gamma}\left(t_{c}, E_{0}, \varphi\right)$ at $80 \mathrm{MeV}$.

Both GCR and SEP fluxes outside the satellite are converted to the corresponding data inside the satellite by using the following equation:

$$
\begin{aligned}
& \Phi_{\mathrm{GCR} \text { or SEP }, S_{\mathrm{in},}, i}\left(t_{\mathrm{c}}, E_{i}\right)= \\
& \sum_{j} \int \Phi_{\mathrm{GCR} \text { or SEP }, S_{\text {out } j} j}\left(t_{c}, E_{j}\right) R_{S, j i}\left(E_{j}, E_{i}\right) \mathrm{d} E_{j}
\end{aligned}
$$

where $E_{j}$ should be replaced with $E_{0}$ for the SEP case. Then, the dose rates whose response function is included in the database of $R_{D, i}\left(E_{i}\right)$ can be estimated in real time by using the following equation:

$$
D_{\mathrm{GCR} \text { or SEP }}\left(t_{\mathrm{c}}\right)=\sum_{i} \int \Phi_{\mathrm{GCR} \text { or SEP }, S_{\mathrm{in}}, i}\left(t_{\mathrm{c}}, E_{i}\right) R_{\mathrm{D}, i}\left(E_{i}\right) \mathrm{d} E_{i}
$$

For forecasting the dose rates at a certain time $t, T_{S, \mathrm{Kp}, j}\left(t, E_{j}\right)$ and $P_{S, \mathrm{Kp}, \theta_{t}}\left(t, E_{0}, \varphi\right)$ are calculated based on the expected satellite location at that time deduced from the TLE data. Then, Equation (1) is replaced by

$$
\begin{aligned}
& \Phi_{\mathrm{SEP}, S_{\text {out }}}\left(t, t_{c}, E_{0}\right)= \\
& \quad \int N_{0}\left(t_{\mathrm{c}}\right) \Phi_{1 \mathrm{AU}, \mathrm{IP}, \gamma}\left(t-t_{0}, E_{0}, \varphi\right) P_{S, \mathrm{Kp}, \theta_{t}}\left(t, E_{0}, \varphi\right) \mathrm{d} \varphi
\end{aligned}
$$

where $\Phi_{\mathrm{SEP}, S_{\text {out }}}\left(t, t_{c}, E_{0}\right)$ denotes the forecasted SEP fluxes outside the satellite at time $t$, assuming the evaluated four parameters, IP, $\theta_{t}, \gamma$ and $N_{0}$, remain stable after current time $t_{c}$. Finally, the forecasted GCR and SEP fluxes inside the satellite, as well as corresponding dose rates, are determined in the same manner as in Equations (2) and (3). 
Table 1. List of experimental data used for validating WASAVIES-EO.

\begin{tabular}{|c|c|c|c|c|}
\hline Quantity & Instrument & Satellite & Date \& GLE & Reference \\
\hline Absorbed dose rate in silicon & DOSTEL & Columbus module of ISS & $\begin{array}{l}\text { 10-11 Sep. } 2017 \\
\text { GLE } 72\end{array}$ & Berger et al. (2018) \\
\hline Proton flux above $100 \mathrm{MeV}$ & MEPED & $\begin{array}{l}\text { NOAA-15, 18, } 19 \\
\text { POES }\end{array}$ & $\begin{array}{l}\text { 10-11 Sep. } 2017 \\
\text { GLE } 72\end{array}$ & Evans and Greer (2000) \\
\hline
\end{tabular}

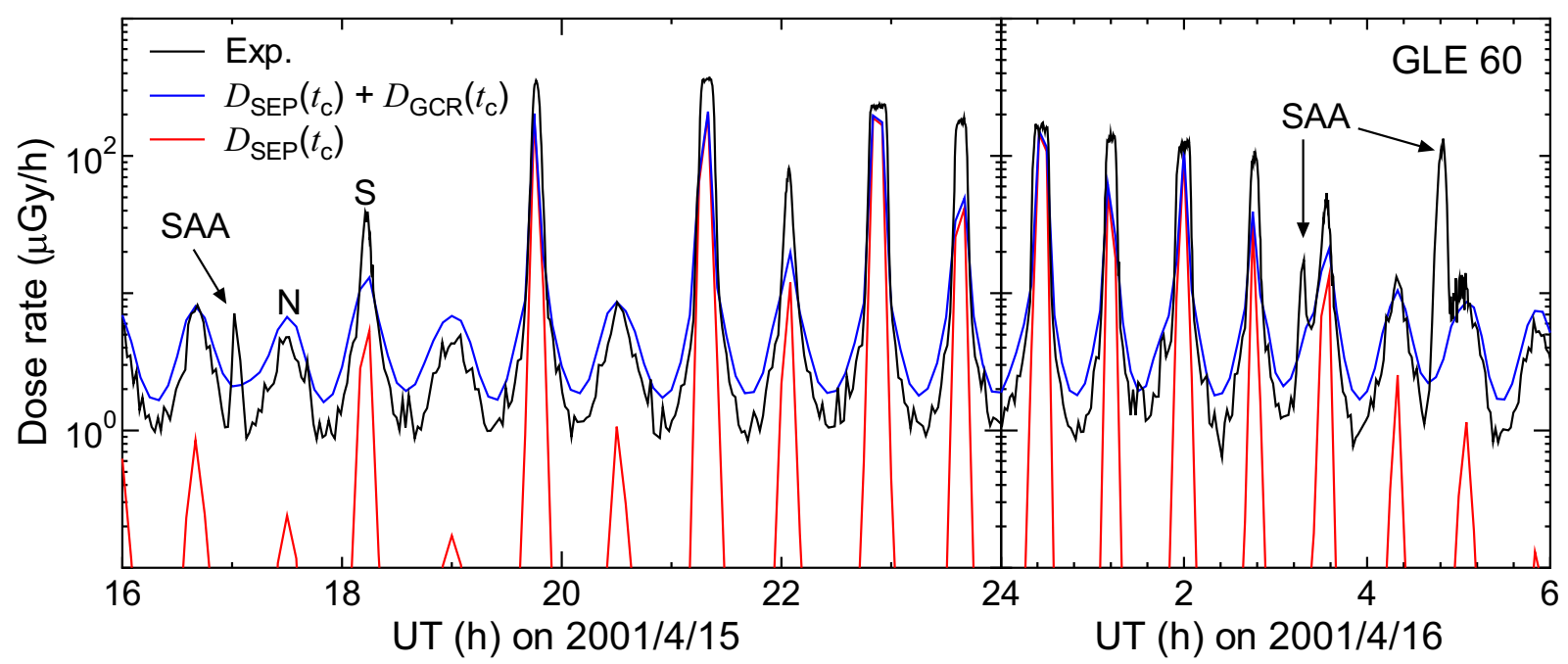

Fig. 3. Comparison between dose rates measured using DOSTEL in US Laboratory of ISS during GLE 60 and corresponding data calculated using WASAVIES-EO. The peaks are alternatively attributable to the northern (N) and southern (S) passages of ISS except for the SAA peaks.

\section{Results and discussion}

The accuracy of WASAVIES-EO was examined using four sets of experimental data, which are listed in Table 1. The absorbed doses in silicon measured by DOSTEL and Liulin-5 in MATROSHKA-R were calculated using their response functions, $R_{D, i}\left(E_{i}\right)$, as described before. However, information about the shielding configurations around those detectors is not available. Thus, in this study, $R_{S, j i}\left(E_{j}, E_{i}\right)$ of the pressurized module of Kibo was substituted with that of the modules on which the detectors were actually loaded during the GLEs. For reproducing the high-energy proton fluxes measured by Medium Energy Proton and Electron Detector (MEPED) loaded on POES, we simply integrated the calculated proton fluxes on their orbits, $\Phi_{\mathrm{GCR}, S_{\text {out }}}\left(t_{c}, E_{0}\right)$ and $\Phi_{\mathrm{SEP}, S_{\text {out }}}\left(t_{c}, E_{0}\right)$, for energies higher than $100 \mathrm{MeV}$.

Figures 3-5 show a comparison of the absorbed dose rates measured in ISS with the corresponding data obtained from WASAVIES-EO. The irregular peaks observed in the measured dose rates can be ascribed to the passage of ISS through the South Atlantic Anomaly (SAA), which cannot be reproduced by our calculation because we did not consider the contribution of TP. Except for those peaks, the measured dose rates oscillated with a period of approximately $50 \mathrm{~min}$ because ISS approaches the polar regions closely with that interval.
The measured dose rates were mostly between 1 and $8 \mu \mathrm{Gy} \mathrm{h}^{-1}$, but they were occasionally considerably higher during GLEs, especially GLE 60 and 72.

Agreements between the measured and calculated dose rates are generally satisfactory, except for the SAA peaks, indicating the validity of WASAVIES-EO for calculating the GCR and SEP dose rates. However, WASAVIES-EO underestimated and overestimated the measured dose rates at the peaks of GLE 60 and 72, respectively. These discrepancies can mostly be ascribed to the use of $R_{S_{j} j i}\left(E_{j}, E_{i}\right)$ of the pressurized module of Kibo instead of that of the modules on which the detectors were loaded. The use of an empirical geomagnetic field model T89 in calculating $P_{S, K p, \theta_{t}}\left(t, E_{0}, \varphi\right)$ might be another cause of the discrepancies because they are more apparently observed for a certain hemisphere - during the northern and southern passages for GLE 60 and 72, respectively. In addition, extrapolation of the SEP fluxes down to $10 \mathrm{MeV}$ by using the same power index at $80 \mathrm{MeV}$ may have led to the overestimation observed at the peaks of GLE 72 because the SEP spectrum generally hardens with decreasing energy (Mertens et al., 2010; Mewaldt et al., 2005). Note that the assumption of the softer spectrum results in overestimation of the lower-energy SEP fluxes in our system because the model parameters were determined to reproduce the observables related to the high-energy SEP fluxes, that is, the count 


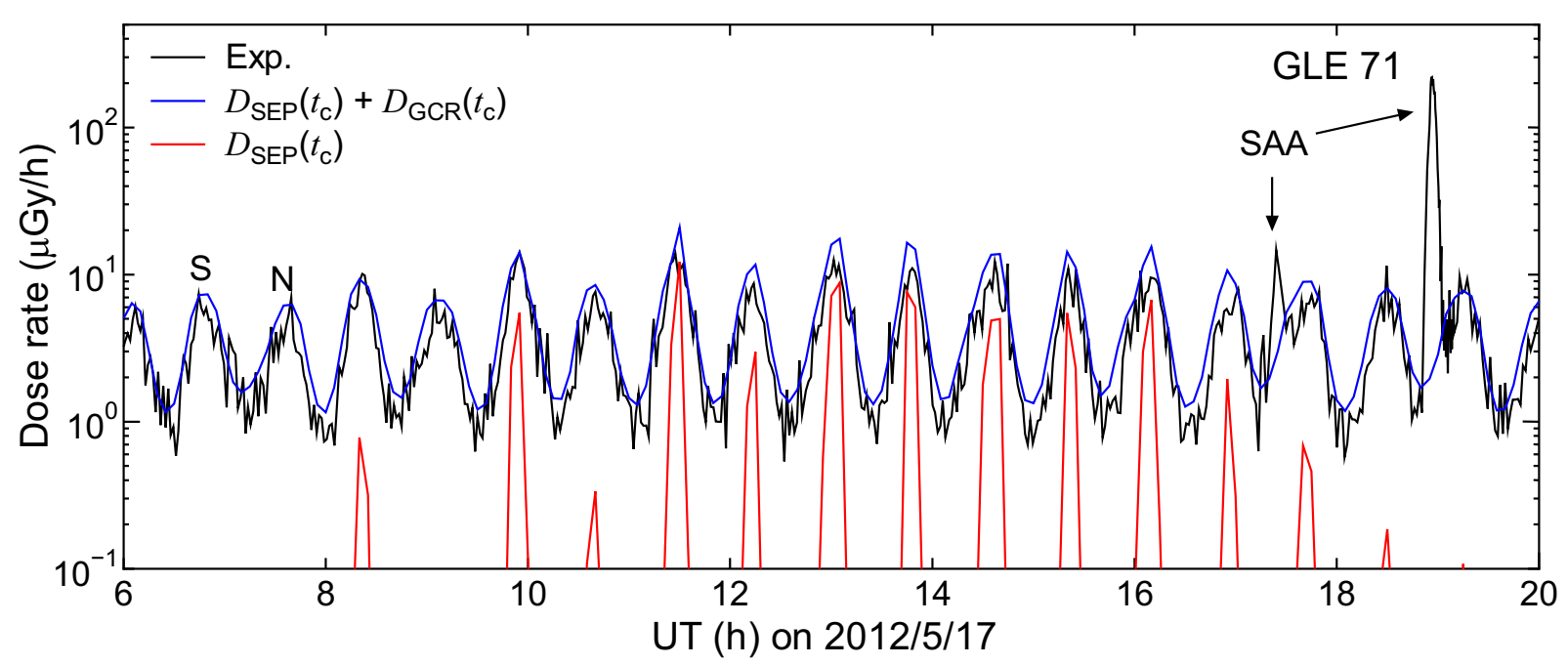

Fig. 4. Comparison between dose rates measured using Liulin-5 in MATROSHKA-R in MIM1 module of ISS during GLE 71 and corresponding data calculated using WASAVIES-EO. The peaks are alternatively attributable to the northern $(\mathrm{N})$ and southern $(\mathrm{S})$ passages of ISS except for the SAA peaks.

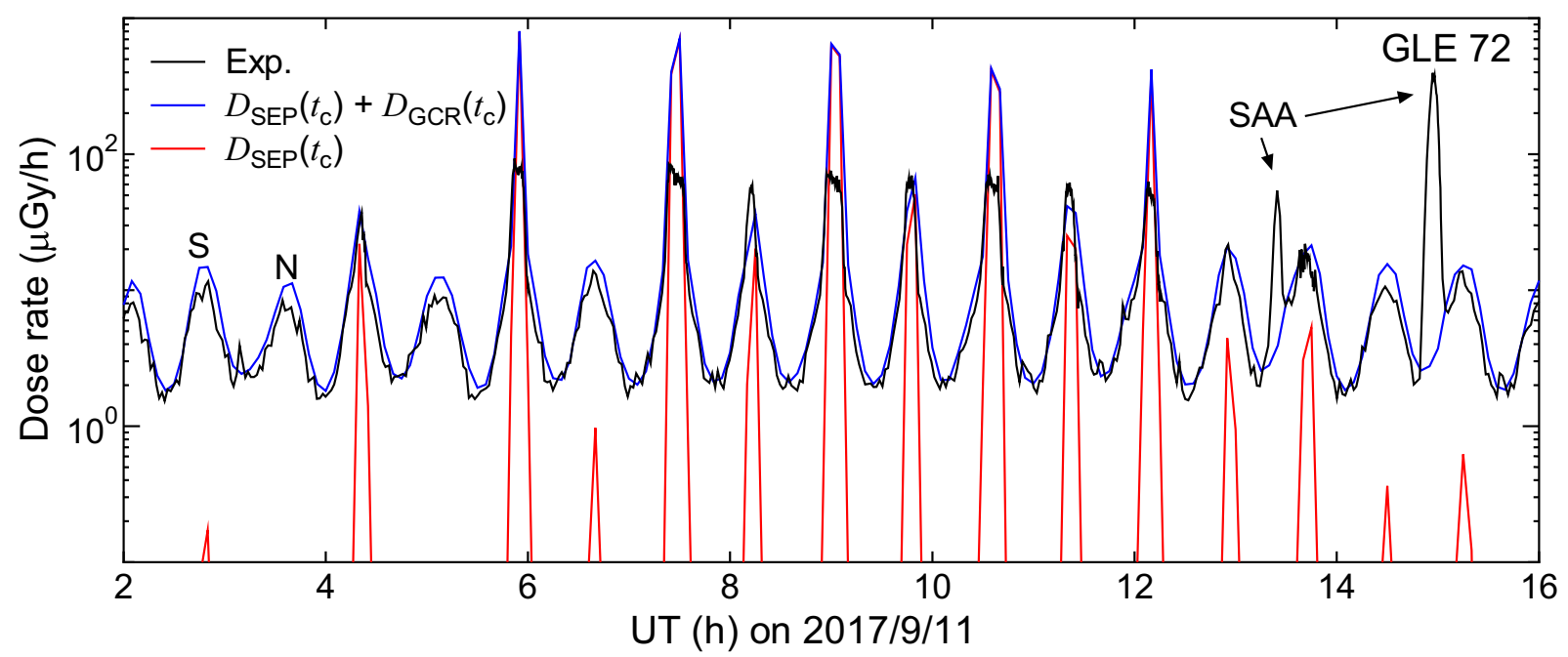

Fig. 5. Comparison between dose rates measured using DOSTEL in Columbus module of ISS during GLE 72 and corresponding data calculated using WASAVIES-EO. The peaks are alternatively attributable to the northern $(\mathrm{N})$ and southern (S) passages of ISS except for the SAA peaks.

rates of the NMs and the GOES proton fluxes higher than $100 \mathrm{MeV}$.

Figure 6 shows a comparison between the proton fluxes higher than $100 \mathrm{MeV}$ measured by POES during GLE 72 and the corresponding data obtained using WASAVIES-EO. The data measured by the three satellites, NOAA-15, -18 , and -19 , were downloaded from the Solar-Terrestrial Physics site of NOAA (https://www.ngdc.noaa.gov/stp/satellite/poes/). The corresponding data measured by GOES are also plotted in each graph. Similar to the absorbed dose rates shown in Figures 3-5, the GCR and SEP fluxes oscillate with a period of approximately $50 \mathrm{~min}$, while irregular TP peaks are observed owing to the complex structure of the radiation belt. Except for the
TP peaks, WASAVIES-EO can reproduce the measured data fairly well, indicating the validity of WASAVIES-EO not only to ISS but also other satellites. However, the WASAVIES-EO overestimated the peak SEP fluxes in the early stages of the GLE, where the calculated fluxes agreed with the data observed by GOES instead of POES. The reason for causing this discrepancy is currently under investigation.

Figure 7 shows a comparison between the organ dose and the dose-equivalent rates for RBM of the reference male phantom inside ISS during GLE 69 and 70, as calculated using WASAVIES-EO, and the effective dose rates at the conventional flight altitude $(12 \mathrm{~km})$ above the South Pole, as calculated using WASAVIES. It is evident from the graphs that the dose 


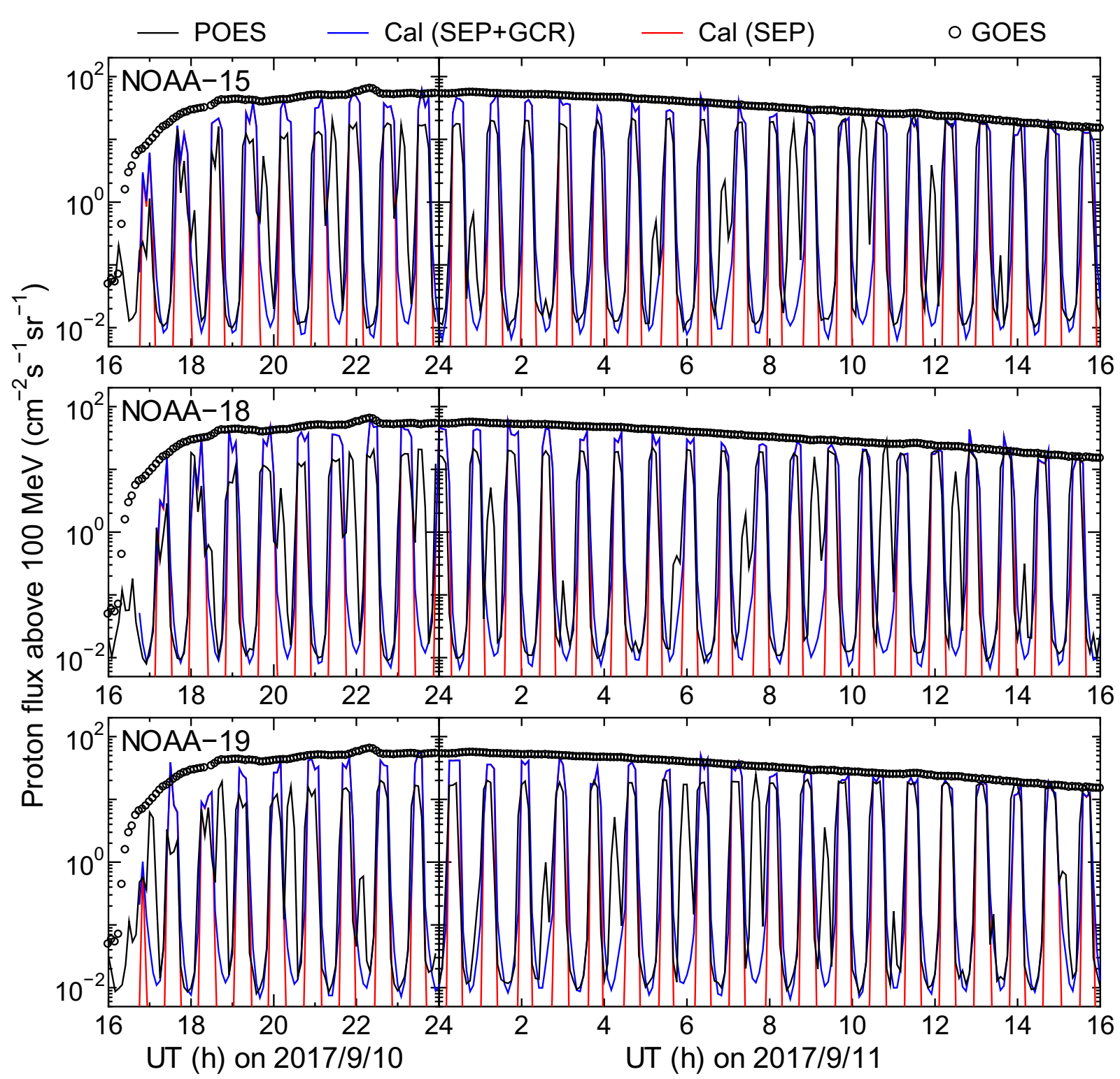

Fig. 6. Comparison between proton fluxes higher than $100 \mathrm{MeV}$ measured using POES during GLE 72 and corresponding data calculated using WASAVIES-EO. The data measured using GOES are plotted in each panel.

rates inside ISS increased in the late and early stages of GLE 69 and 70, respectively. This is because the orbital inclination of ISS is approximately $52^{\circ}$, and the SEP fluxes do not always increase when ISS approaches the polar regions closely because of lower L values on the orbit. Thus, the doses to ISS astronauts depend significantly on the timing of flare onset; GLE 70 occurred nearly at the worst timing in terms of the SEP exposure to astronauts because ISS passed through higher L-value locations up to around six during the GLE peak.

Table 2 summarizes the total doses calculated by integrating the data shown in Figure 7 over $24 \mathrm{~h}$ from the flare onset. The mean quality factors are approximately 2.3 and 1.6 for the GCR and SEP doses, respectively. It should be mentioned that the calculated RBM doses as well as their mean quality factors are lower than the corresponding data measured by small detectors such as DOSTEL because of the self-shielding of human body. In addition, both GLEs occurred during Forbush decreases of earlier events, and thus, the GCR dose rates during the GLEs were slightly suppressed in comparison to the solar quiet condition.

Owing to the worst timing of occurrence of GLE 70 as discussed before, the total doses inside ISS during the event are higher than those during GLE 69, despite GLE 69 has been the largest event that has occurred in the 21 st century. The dose equivalents in ISS are comparable to the corresponding effective doses at the flight altitude instead of the lower shielding thickness of ISS than that of the atmosphere, owing to the protection provided by the magnetosphere. If ISS were outside the magnetosphere during the entire span of GLE 69, the total dose equivalent would have been approximately $8 \mathrm{mSv}$. Note that the 

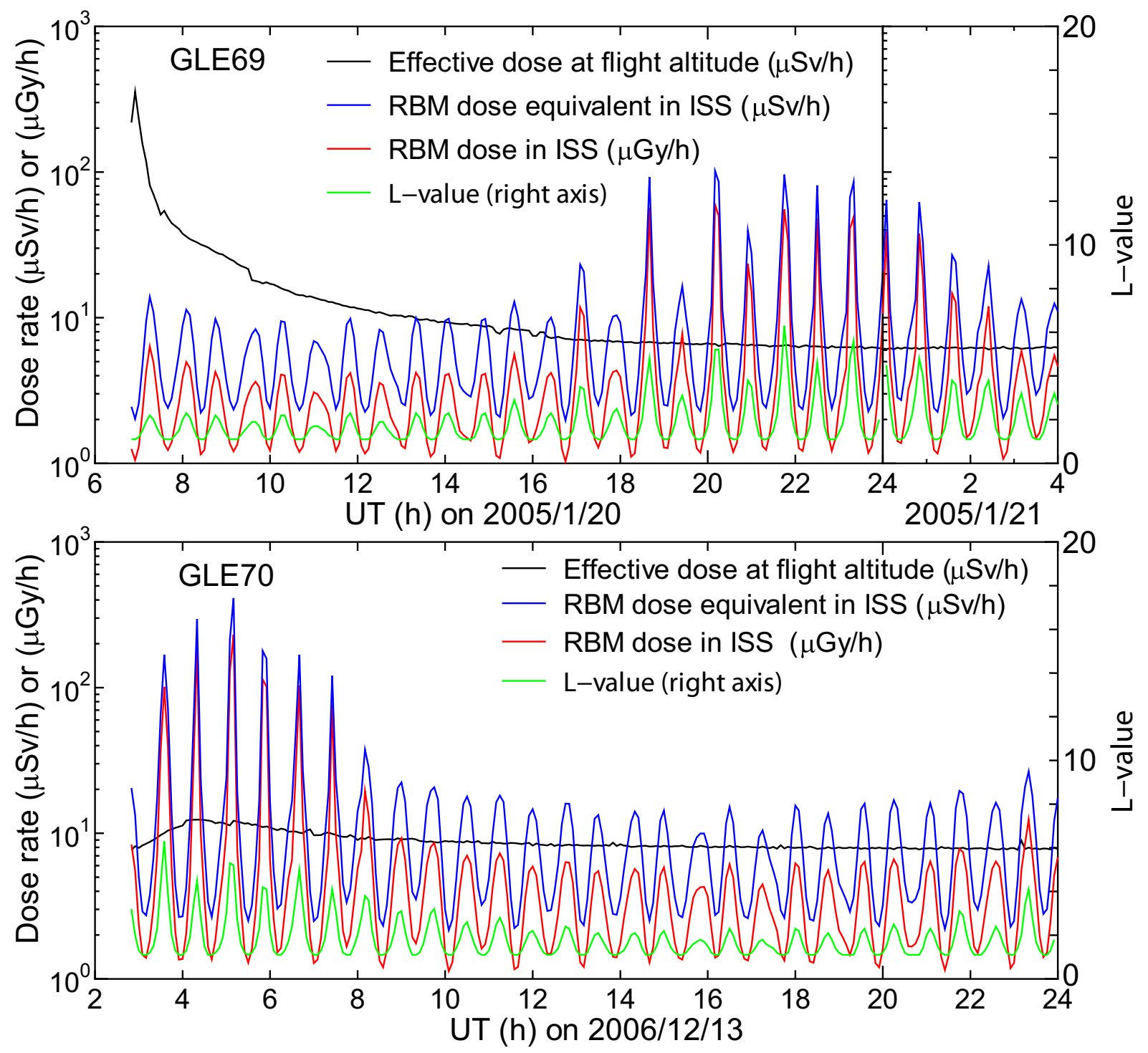

Fig. 7. Comparison between organ dose and dose equivalent rates for red-bone marrow (RBM) of the reference male phantom inside ISS during GLE 69 and 70 calculated using WASAVIES-EO and effective dose rates at conventional flight altitude (12 km) above South Pole calculated using WASAVIES. L-value at the ISS location is also plotted.

Table 2. Total doses calculated by integrating the data shown in Fig. 7 over $24 \mathrm{~h}$ from flare onset.

\begin{tabular}{|c|c|c|c|c|c|c|}
\hline & \multicolumn{3}{|c|}{ GLE 69} & \multicolumn{3}{|c|}{ GLE 70} \\
\hline & GCR & SEP & Total & GCR & SEP & Total \\
\hline RBM dose in ISS ( $\mu \mathrm{Gy}$ ) & 63.3 & 48.0 & 111 & 93.5 & 94.6 & 188 \\
\hline Effective dose at $12 \mathrm{~km}(\mu \mathrm{Sv})$ & 113 & 297 & 410 & 184 & 88.3 & 272 \\
\hline
\end{tabular}

total effective doses shown in the table are expected to be considerably higher than the values actually received by an aircrew because it is unrealistic to stay at the flight altitude for $24 \mathrm{~h}$.

Figure 8 shows the forecasted dose rates by WASAVIESEO and WASAVIES for the same condition as in Figure 7, where the forecast started $20 \mathrm{~min}$ after GLE detection, that is, at 7:10 UT on $2005 / 1 / 20$ and $3: 10$ UT on $2006 / 12 / 13$ for GLE 69 and 70, respectively. Agreement between the data shown in Figures 7 and 8 seems to be reasonable, especially in terms of their temporal structures. This tendency demonstrates the usefulness of WASAVIES-EO for future mission operation because it can predict the approximate time to take 

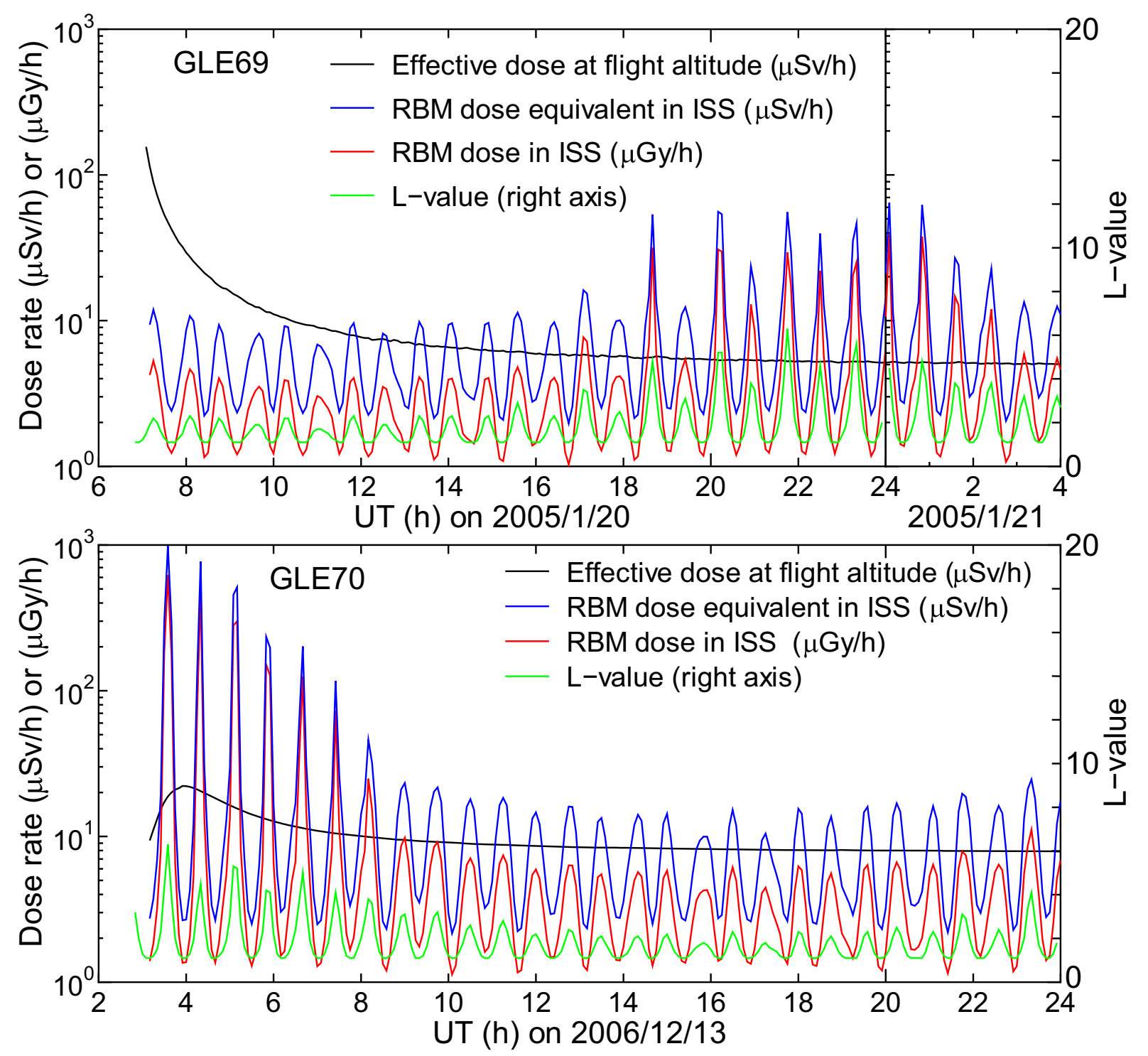

Fig. 8. Same as Fig. 7 but forecasted 20 min after GLE detection, that is, at 7:10 UT on 2005/1/20 and 3:10 UT on 2006/12/13 for GLE 69 and 70 , respectively.

adequate actions against SEP exposure, such as sheltering astronauts in well-shielded locations in the spacecraft. However, the absolute values of the forecasted dose rates at the peaks of SEP exposure are smaller and larger than the corresponding data shown in Figure 7 for GLE 69 and 70, respectively. This discrepancy can be ascribed to the gradual changes in the best-fit parameters evaluated using WASAVIES, as discussed in our previous paper (Sato et al., 2018b).

\section{Conclusions}

A physics-based warning system for aviation exposure to SEP, WASAVIES, was extended to be capable of nowcasting and forecasting the GCR and SEP fluxes not only in the atmosphere but also in the magnetosphere and ionosphere during a large SPE associated with a GLE. The extended version, called
WASAVIES-EO, can calculate the GCR and SEP fluxes outside a satellite based on the TLE data of the satellite. In addition, the system can estimate the organ dose and dose-equivalent rates of astronauts in ISS, considering the shielding effect of ISS, as evaluated by PHITS coupled with a virtual ISS model. The accuracy of WASAVIES-EO was well validated by the dose rates measured in ISS as well as by the high-energy proton fluxes observed by POES during large GLEs that have occurred in the 21st century. Agreement between the nowcast and the forecast dose rates in ISS, especially in terms of their temporal structures, suggested the usefulness of the system for future mission operations. A function to calculate the TP fluxes should be implemented before developing an operational WASAVIES-EO system for practical use.

Two issues should be addressed for the further improvements of WASAVIES-EO. One is the limitation of 1-D simulation to solve the focused transport equation because the 
assumption of well-connected magnetic field geometry is not always the case for GLEs. Energetic particles occasionally pass through complex 3-D dynamic structures as caused by the multiple occurrences of CMEs (Meyer et al., 1956; Richardson et al., 1991). We therefore need to properly incorporate the results from 3-D focused transport simulations, allowing the complexity of the background interplanetary magnetic field. The other issue is the inaccuracy in dose estimation due to the simple power-law extrapolation of the primary SEP fluxes below $80 \mathrm{MeV}$. It is known that the time evolution of CMEs associated interplanetary shocks and the magnetic field connectivity to the shocks make the energy spectra of SEP below $100 \mathrm{MeV}$ very complicated (Luhmann et al., 2017). For nowcast, we can utilize the real-time proton data measured by satellites, but we need to study and model the complex behavior of such low-energy SEPs for forecast. For tackling both issues, realistic 3-D magnetic field structures of the inner heliosphere, as obtained from the 3-D magnetohydrodynamic simulations of CMEs such as SUSANOO-CME model (Shiota \& Kataoka, 2016), will play essential role. The replacement of the geomagnetic field model T89 by its updated model (Tsyganenko \& Andreeva, 2015) is also desirable.

Acknowledgements. We wish to thank Dr. J. Semkova at Bulgarian Academy of Sciences, Dr. V. Shurshakov at Russian Academy of Sciences, and Dr. D. Matthiä, Dr. T. Berger, and Dr. G. Reitz at DLR for providing the experimental data of Liulin-5 and DOSTEL used for the validation of WASAVIES-EO. We acknowledge the NMDB database founded under the European Union's FP7 programme (contract no. 213007). This work was partially supported by JSPS grant KAKENHI (26106006, 15H05813, 15H05815, $16 \mathrm{H} 01180,17 \mathrm{~K} 05671)$. The editor thanks two anonymous referees for their assistance in evaluating this paper.

\section{References}

Aghara SK, Sriprisan SI, Singleterry RC, Sato T. 2015. Shielding evaluation for solar particle events using MCNPX, PHITS and OLTARIS codes. Life Sci Space Res 4: 79-91. DOI: 10.1016/j. 1ssr.2014.12.003.

Berger T, Matthiä D, Burmeister S, Rios R, Lee K, Semones E, Hassler DM, Stoffle N, Zeitlin C. 2018. The Solar Particle Event on 10 September 2017 as observed on-board the International Space Station (ISS). Space Weather 16: 1173-1189. DOI: 10.1029/ 2018 SW001920.

Evans DS, Greer MS. 2000. Polar orbiting environmental satellite space environment monitor - 2 Instrument descriptions and archive data documentation, NOAA Technical Memorandum OAR SEC 93, Boulder, Colorado.

Ginet GP, O'Brien TP, Huston SL, Johnston WR, Guild TB, et al. 2013. AE9, AP9 and SPM: New models for specifying the trapped energetic particle and space plasma environment. Space Sci Rev 179: 579-615. DOI: $10.1007 / \mathrm{s} 11214-013-9964-y$.

Heynderickx D, Quaghebeur B, Wera J, Daly EJ, Evans HDR. 2004. New radiation environment and effects models in the European Space Agency's Space Environment Information System (SPENVIS). Space Weather 2: S10S03. DOI: 10.1029/2004sw000073.

Hu S, Zeitlin C, Atwell W, Fry D, Barzilla JE, Semones E. 2016. Segmental interpolating spectra for solar particle events and in situ validation. Space Weather 14: 742-753. DOI: 10.1002/ 2016SW001476.

ICRP. 2010. Conversion coefficients for radiological protection quantities for external radiation exposures, ICRP Publication 116. Ann ICRP 40: 2-5.

ICRP. 2013. Assessment of radiation exposure of astronauts in space, ICRP Publication 123. Ann ICRP 42: 1-339.

Jiggens P, Heynderickx D, Sandberg I, Truscott P, Raukunen O, Vainio R. 2018. Updated model of the solar energetic proton environment in space. J Space Weather Space Clim 8: A31. DOI: 10.1051/Swsc/2018010.

Kataoka R, Sato T, Kubo Y, Shiota D, Kuwabara T, Yashiro S, Yasuda H. 2014. Radiation dose forecast of WASAVIES during ground-level enhancement. Space Weather 12: 380-386. DOI: 10.1002/2014sw001053.

Kataoka R, Sato T, Miyake S, Shiota D, Kubo Y. 2018. Radiation dose nowcast during the ground level enhancement on 10-11 September 2017. Space Weather 16: 917-923. DOI: 10.1029/ 2018SW001874.

Kubo Y, Kataoka R, Sato T. 2015. Interplanetary particle transport simulation for warning system for aviation exposure to solar energetic particles. Earth Planets Space 67: 117. DOI: 10.1186/ S40623-015-0260-9.

Luhmann JG, Mays ML, Odstrcil D, Li Y, Bain H, et al. 2017. Modeling solar energetic particle events using ENLIL heliosphere simulations. Space Weather 15: 934-954. DOI: 10.1002/ 2017 SW001617.

Matthiä D, Berger T, Mrigakshi AI, Reitz G. 2013. A ready-to-use galactic cosmic ray model. Adv Space Res 51: 329-338. DOI: 10.1016/j.asr.2012.09.022.

Matthiä D, Meier M, Berger T. 2018. The solar particle event on 10-13 September 2017 - Spectral reconstruction and calculation of the radiation exposure in aviation and space. Space Weather $\mathbf{1 6}(\mathbf{8})$ : 977-986. DOI: 10.1029/2018SW001921.

Mertens C, Slaba T, Hu S. 2018. Active dosimeter-based estimate of astronaut acute radiation risk for real-time solar energetic particle events. Space Weather 16(9): 1291-1316. DOI: 10.1029/ 2018SW001971.

Mertens CJ, Kress BT, Wiltberger M, Blattnig SR, Slaba TS, Solomon SC, Engel M. 2010. Geomagnetic influence on aircraft radiation exposure during a solar energetic particle event in October 2003. Space Weather 8: S03006. DOI: 10.1029/2009sw000487.

Mewaldt RA, Cohen CMS, Labrador AW, Leske RA, Mason GM, et al. 2005. Proton, helium, and electron spectra during the large solar particle events of October-November 2003. J Geophys Res 110: A9. DOI: $10.1029 / 2005 j a 011038$.

Meyer P, Parker EN, Simpson JA. 1956. Solar cosmic rays of February, 1956 and their propagation through interplanetary space. Phys Rev 104: 768.

Miyake S, Kataoka R, Sato T. 2017. Cosmic ray modulation and radiation dose of aircrews during the solar cycle 24/25. Space Weather 15: 589-605. DOI: 10.1002/2016SW001588.

Nymmik RA, Panasyuk MI, Suslov AA. 1996. Galactic cosmic-ray flux simulation and prediction, near-earth radiation environment including time variations and secondary radiation. Adv Space Res 17: $19-30$.

O'Neill PM, Golge S, Slaba T. 2014. Badhwar-O'Neill 2014 galactic cosmic ray flux model description. NASA/TP-2015-218569.

Reitz G, Beaujean R, Benton E, Burmeister S, Dachev T, Deme S, Luszik-Bhadra M, Olko P. 2005. Space radiation measurements on-board ISS - The DOSMAP experiment. Radiat Prot Dosim 116: 374-379. DOI: $10.1093 / \mathrm{rpd} /$ nci262. 
Richardson IG, Cane HV, Vonrosenvinge TT. 1991. Prompt arrival of solar energetic particles from far-eastern events - The role of large-scale interplanetary magnetic-field structure. J Geophys Res 96: 7853-7860. DOI: $10.1029 / 91 \mathrm{ja} 00379$.

Sato T. 2015. Analytical model for estimating terrestrial cosmic ray fluxes nearly anytime and anywhere in the world: Extension of PARMA/EXPACS. PLoS One 10: e0144679. DOI: 10.1371/ journal.pone.0144679.

Sato T. 2016. Analytical model for estimating the zenith angle dependence of terrestrial cosmic ray fluxes. PLoS One 11: e0160390. DOI: 10.1371/journal.pone.0160390.

Sato T, Iwamoto Y, Hashimoto S, Ogawa T, Furuta T, et al. 2018a. Features of particle and heavy ion transport code system PHITS Version 3.02. J Nucl Sci Technol 55: 684-690. DOI: 10.1080/ 00223131.2017.1419890.

Sato T, Kataoka R, Shiota D, Kubo Y, Ishii M, Yasuda H, Miyake S, Park I, Miyoshi Y. 2018b. Real-time and automatic analysis program for WASAVIES: Warning system for aviation exposure to solar energetic particles. Space Weather 16: 924-936. DOI: 10.1029/2018SW001873.

Sato T, Kataoka R, Yasuda H, Yashiro S, Kuwabara T, Shiota D, Kubo Y. 2014. Air shower simulation for WASAVIES: Warning system for aviation exposure to solar energetic particles. Radiat Prot Dosim 161: 274-278. DOI: 10.1093/Rpd/ Nct332.

Sato T, Nagamatsu A, Ueno H, Kataoka R, Miyake S, Takeda K, Niita K. 2018c. Comparison of cosmic-ray environments on Earth, Moon, Mars, and spacecraft using PHITS. Radiat Prot Dosim 180: 146-149. DOI: 10.1093/rpd/ncx192.
Semkova J, Dachev T, Koleva R, Bankov N, Maltchev S, Benghin V, Shurshakov V, Petrov V. 2014. Observation of radiation environment in the International Space Station in 2012March 2013 by Liulin-5 particle telescope. J Space Weather Space Clim 4: A32. DOI: $10.1051 / \mathrm{Swsc} / 2014029$.

Shiota D, Kataoka R. 2016. Magnetohydrodynamic simulation of interplanetary propagation of multiple coronal mass ejections with internal magnetic flux rope (SUSANOO-CME). Space Weather 14: 56-75. DOI: 10.1002/2015SW001308.

Slaba TC, Blattnig SR. 2014. GCR environmental models I: Sensitivity analysis for GCR environments. Space Weather 12: 217-224. DOI: 10.1002/2013SW001025.

Townsend LW, Adams JH, Blattnig SR, Clowdsley MS, Fry DJ, et al. 2018. Solar particle event storm shelter requirements for missions beyond low Earth orbit. Life Sci Space Res 17: 32-39. DOI: 10.1016/j.1ssr.2018.02.002.

Tsyganenko NA. 1989. A magnetospheric magnetic-field model with a warped tail current sheet. Planet Space Sci 37: 5-20. DOI: 10.1016/0032-0633(89)90066-4.

Tsyganenko NA, Andreeva VA. 2015. A forecasting model of the magnetosphere driven by an optimal solar wind coupling function. J Geophys Res 120: 8401-8425. DOI: 10.1002/2015JA021641.

Tylka AJ, Adams JH, Boberg PR, Brownstein B, Dietrich WF, et al. 1997. CREME96: A revision of the cosmic ray effects on microelectronics code. IEEE Trans Nucl Sci 44: 2150-2160. DOI: 10.1109/23.659030.

Xapsos MA, Summers GP, Barth JL, Stassinopoulos EG, Burke EA. 1999. Probability model for worst case solar proton event fluences. IEEE Trans Nucl Sci 46: 1481-1485. DOI: 10.1109/23.819111.

Cite this article as: Sato T, Kataoka R, Shiota D, Kubo Y, Ishii M, et al. 2019. Nowcast and forecast of galactic cosmic ray (GCR) and solar energetic particle (SEP) fluxes in magnetosphere and ionosphere - Extension of WASAVIES to Earth orbit. J. Space Weather Space Clim. 9, A9. 